\title{
Is Positive Gravitational Force Source of Dark Energy?
}

\author{
Branko M. Novakovic
}

\begin{abstract}
As it is well known today, the universe continues to expand, even at an accelerating rate. The main reason for it is an unknown energy called a dark energy. This energy occupies about 68 percents of the total energy in the universe. One of the candidates for the source of dark energy is a cosmological constant $\Lambda$. Meanwhile, recently developed Relativistic Alpha Field (RAF) theory predicts (among the others) that the gravitational force becomes positive (repulsive) if $\left(G M / r c^{2}\right)>1$, that could be a source of a dark energy. Here we derived equations of the universe motion using field parameters from RAF theory. These equations show that positive (repulsive) gravitational force really can produce an accelerating rate of the universe motion and therefore could be a candidate for the source of a dark energy. In that sense a dark energy can be seen as a positive (repulsive) gravitational energy. In order to compare the RAF theory solution of the universe motion with one of the existing solution, here we also derived related solution with the cosmological constant $\Lambda$.
\end{abstract}

Index Terms-Relativistic alpha field theory, Equations of the universe motion, Dark energy problem solution, Positive (repulsive) gravitational force

\section{INTRODUCTION}

As it is well known today, the universe continues to expand, even at an accelerating rate. The main reason for it is an unknown energy called a dark energy. This energy occupies about 68 percent of the total energy in the universe. There exist more dynamic models of the universe motion. The most known is the model that has been developed independently by Alexander Friedmann [1,2], Georges Lemaitre [3,4], Howard Percy Robertson [5,6,7] and Arthur Geoffrey Walker [8]. Therefore, it has been named Friedmann - Lemaitre Robertson - Walker (FLRW) model. In the literature one can find also the names Friedmann - Robertson - Walker (FRW), or Robertson - Walker (RW), or Friedmann - Lemaitre (FL) model. In the modern cosmology it is also called Standard Model (ST) [9]. The FLRW model describes a homogeneous, isotropic expanding or contracting universe. The general metric has been introduced on the assumption that the universe geometric properties are homogeneous and isotropic, i.e. the Cosmological Principle is valued. Empirically, this is justified on scales larger than $\sim 100 \mathrm{Mpc}$. They also used the Einstein field equations [10,11] for derivation of the "scale factor" of the universe as a function of time. The FLRW model is well described in the references $[1-9,12-18]$.

Branko Novakovic, FSB - University of Zagreb, Luciceva 5, P.O.B. 509 , 10000 Zagreb, Croatia.
The all have been started by Einstein, who proposed a static model of the universe, based on the cosmological constant $\Lambda[10,11]$. Then, Edwin Hubble discovered in 1929, that our universe is expanding. Georges Lemaitre proposed his theory in 1931, that the universe is born from "praatom" and George Gamov introduced in 1948 the new theory about "praatom" , the Big Bang theory. In 1979 Alan Guth proposed the inflationary model of the universe, with explanation why it is considered geometrically flat [19]. Today the universe continues to expand, and at an accelerating rate [20]. Caldwell, Dave and Steinhardt proposed the quintessence mechanism [21]. In the Friedmann - Lemaitre - Robertson Walker cosmologies [1-8], both the cosmological constant and the quintessence approaches are added to the standard Cold Dark Matter (CDM) model, what is resulted with known $\Lambda \mathrm{CDM}$ and QCDM models, respectively. Parker and Raval proposed a new Vacuum Cold Dark Matter (VCDM) model [22]. Steinhard and Turok offered the Cyclic Universe model, as a radical new cosmological scenario [23]. Recently, some new aspects of the universe motion are presented in the references [24-36]. In that context some kind of a phantom scalar field as the source of the universe motion is discussed among the others in the reference [37]. The main problem of the all models of the universe motion is to answer (among the others) to the important questions of the cosmology [23]: what occurred at the initial singularity?, how old is the universe?, how big is the universe? and what is its ultimate fate? One of the new approaches to the derivation of the velocity and acceleration equations of the universe motion for $\Lambda=$ constant and $\Lambda=f(r)$ has been proposed in [35]. The solution that $\Lambda=f(r)$ has the important influence to the velocity and acceleration equations of the universe motion. Recently, a new method for testing of the Cosmological Principle by using an isotropic blackbody cosmic microwave background radiation, as evidence for a homogeneous universe, has been presented in the reference [38].

Today we know that the universe continues to expand, even at an accelerating rate. This acceleration is caused by an unknown energy called a dark energy. But we do not know the source of dark energy. One of the candidates for the source of dark energy is a cosmological constant $\Lambda$. Meanwhile, recently developed Relativistic Alpha Field (RAF) theory $[39,40,41]$ predicts (among the others) that the gravitational force becomes positive (repulsive) if $\left(G M / r c^{2}\right)$ > 1 , that could be a source of a dark energy. Here we derived equations of the universe motion using field parameters from RAF theory. These equations show that positive (repulsive) gravitational force really can produce an accelerating rate of the universe motion and therefore could be a candidate for the source of a dark energy. In that sense a dark energy can be seen as a positive (repulsive) gravitational energy. In order to 
compare the RAF theory solution of the universe motion with one of the existing solution, here we also derived related solution with the cosmological constant $\Lambda$.

\section{FRIEDMANN - LEMAITRE - ROBERTSON - WALKER METRIC MODEL}

The FLRW model describes a homogeneous, isotropic expanding or contracting universe. The general metric has been introduced on the assumption that the universe geometric properties are homogeneity and isotropy, i.e. the Cosmological Principle is valued. Empirically, this is justified on scales larger than $\sim 100 \mathrm{Mpc}$. They also used the Einstein field equations [10,11] for derivation of the "scale factor" of the universe as a function of time. The question of whether the universe is spatially homogenous and isotropic on the largest scales is of fundamental importance to cosmology but has not yet been answered decisively. Recently, a new method for testing of the Cosmological Principle by using an isotropic blackbody cosmic microwave background radiation, as evidence for a homogeneous universe, has been proposed in the reference [38].

General metric that satisfies the homogeneity and isotropy of the space, as well as the possibility that the special components of the metric can be time dependent, is described by the following line element:

$$
d s^{2}=-c^{2} d \tau^{2}=-c^{2} d t^{2}+\mathbf{\alpha}(t)^{2} d \sum^{2} .
$$

Here $d s$ is the line element, $c$ is the speed of the light in vacuum, $\tau$ is a proper time, $t$ is a coordinate time, $\mathbf{\alpha}(t)$ is a "scale factor" and $d \sum^{2}$ is a three dimensional spatial metric that describes a flat space, or a sphere with constant positive curvature, or a hyperbolic space with constant negative curvature. The term $d \sum^{2}$ is normally written as a function of three spatial coordinates. There are several conventions for describing this spatial metric. In the case of reduced circumference polar coordinates, the spatial metric has the form:

$d \Sigma^{2}=\frac{d r^{2}}{1-\mathrm{K} r^{2}}+r^{2} d \Omega^{2}, d \Omega^{2}=d \theta^{2}+\sin ^{2} \theta d \phi^{2}$.

In the previous relation $\mathrm{K}$ is a constant representing the curvature of the space. Here one should distinguish the two common unit conventions.

In the first one $\mathrm{K}$ may be taken to have unit of length ${ }^{-2}$, in which case $r$ has unit of length and $\alpha(t)$ is unitless. In that case $\mathrm{K}$ is the spatial (Gaussian) curvature of the space at the time when $\mathrm{a}(t)=1$ (i.e. today). Also $\mathrm{r}$ is sometimes called the reduced circumference because it is equal to the measured circumference of a circle, at that value of $r$, centered at the origin, divided by $2 \pi$, like the $r$ of Schwarzschild coordinates. If the shape of the universe is hyperspherical and $R(t)$ is the radius of curvature $\left(R_{0}\right.$ in the present day), then $\alpha(t)=R(t) / R_{0}$. If $\mathrm{K}$ is positive, then the universe is hyperspherical. If $\mathrm{K}$ is zero, then the universe is flat. Finally, if $\mathrm{K}$ is negative, then the universe is hyperbolic.

In the second common unit convention $\mathrm{K}$ may be chosen from the set of numbers $(-1,0,1)$ for negative (an open 3-hyperboloid), zero (flat, i.e. Euclidian space) and positive (closed 3-sphere) curvature, respectively. In that case $r$ is unitless and $\mathrm{\alpha}(t)$ has unit of length. If $\mathrm{K}=+1$, then $\mathrm{\alpha}(t)$ is the radius of curvature of the space that sometimes may be written by $R(t)$. If $\mathrm{K}=0$, then $\mathrm{\alpha}(t)$ may be fixed to any arbitrary positive number at one particular time. If $\mathrm{K}=-1$, then (loosely speaking) one can say that $\mathrm{i} \cdot \mathbf{\alpha}(t)$ is the radius of curvature of the space. In the case of positive curvature the reduced circumference coordinates cover only half of the 3 -sphere. This is a disadvantage of the reduced circumference coordinates.

\section{FRIEDMANN EQATIONS}

Following the assumption that the universe geometric properties are homogeneous and isotropic, i.e. the Cosmological Principle is valid, the Friedmann equations have been first derived by Alexander Friedmann in 1922 [1]. The related equations for negative spatial curvature have been presented by Friedmann in 1924 [2]. He also used the full form of the Einstein's field equations in the General Theory of Relativity that is given by the relation $[10,11]$ :

$R_{\mu \eta}-\frac{1}{2} g_{\mu \eta} R+\Lambda g_{\mu \eta}=\frac{8 \pi G}{c^{4}} T_{\mu \eta}, \mu, \eta=0,1,2,3$.

In this relation $\Lambda$ is the Einstein's cosmological constant, $G$ is the Newton's gravitational constant, $c$ is the speed of the light in a vacuum and $T_{\mu \eta}$ is the energy-momentum tensor. Determination of the time evolution of the scale factor $\alpha(t)$ requires the Einstein's field equations together with a way of calculation of density, $\rho(t)$, such as a cosmological equation of state. If the energy-momentum tensor, $T_{\mu \eta}$, is similarly assumed to be isotropic and homogenous, then the metric (1) has an analytic solution to Einstein's field equations (3) giving the Friedmann equations in the form:

$$
\begin{aligned}
& \left(\frac{\dot{\alpha}}{\alpha}\right)^{2}+\frac{\mathrm{K} c^{2}}{\alpha^{2}}-\frac{\Lambda c^{2}}{3}=\frac{8 \pi G}{3} \rho, \\
& 2 \frac{\ddot{\alpha}}{\alpha}+\left(\frac{\dot{\alpha}}{\alpha}\right)^{2}+\frac{K c^{2}}{\alpha^{2}}-\Lambda c^{2}=-\frac{8 \pi G}{c^{2}} p .
\end{aligned}
$$

Here $\alpha(t)$ is the scale factor with related time derivations $\dot{\alpha}$ and $\ddot{\alpha}$, while $\rho$ and $p$ are fluid mass density and pressure, respectively. The presented equations (4) are the basis of the standard big bang cosmological model including the current $\Lambda \mathrm{CDM}$ model. Following the mentioned assumption that the universe is isotropic and homogenous, the model (4) can be used as a first approximation for the evolution of the real, lumpy universe, because it is simple for calculation. Further more, the models which calculate the lumpiness in the universe can be added to this model as extensions.

The pair of the equations in (4) is equivalent to the following pair of the equations:

$$
\begin{aligned}
& \frac{\dot{\alpha}^{2}+\mathrm{K} c^{2}}{\alpha^{2}}=\frac{8 \pi G \rho+\Lambda c^{2}}{3}, \\
& \quad \frac{\ddot{\alpha}}{\alpha}=-\frac{4 \pi G}{3}\left(\rho+\frac{3 p}{c^{2}}\right)+\frac{\Lambda c^{2}}{3} .
\end{aligned}
$$




\section{International Journal of New Technology and Research (IJNTR) \\ ISSN:2454-4116, Volume-1, Issue-7, November 2015 Pages 06-13}

The first equation in (5) has been derived from the 00 component of the Einstein's field equations (3). On the other side, the second equation in (5) is derived from the trace of the Einstein's field equations (3). It is easy to see that the first equation in (5) can be rewritten into the form of the first equation in (4). The second equation in (5) can be obtained by substitution of the term $(\dot{\alpha} / \alpha)^{2}$ from the first to the second equations in (4). This confirms that the pair of the equations in (4) is equivalent to the related pair of the equations in (5). Some cosmologists call the second equation in (5) as Friedmann acceleration equation and reserve the term Friedmann equation for only the first equation in (5).

Now, one can employ the time derivative of the first equation in (5) and combine it with the second equation in (5). As the result one obtains the new pair of the equations that are also equivalent to the pairs of the equations in (4) and (5):

$$
\begin{aligned}
\dot{\rho}=-3 \frac{\dot{\alpha}}{\alpha} & \left(\rho+\frac{p}{c^{2}}\right), \\
\frac{\ddot{\alpha}}{\alpha} & =-\frac{4 \pi G}{3}\left(\rho+\frac{3 p}{c^{2}}\right)+\frac{\Lambda c^{2}}{3} .
\end{aligned}
$$

Here the special curvature index serving as a constant of integration for the second equation in (6). The first equation in (6) can be derived also from thermo-dynamical consideration and is equivalent to the first law of thermodynamics, assuming that the expansion of the universe is an adiabatic process. This assumption has been implicitly included into the derivation of the Friedmann - Lemaitre Robertson - Walker metric. The second equation in (6) states that both the energy density and the pressure cause the expansion rate of the universe, $\dot{\alpha}$, to decrease. It means that both the energy density and the pressure cause a deceleration in the expansion of the universe. This is the consequence of gravitation, including that pressure is playing a similar role to that of energy (or mass) density. This is, of course, in accordance with the principles of general relativity. On the other side, the cosmological constant, $\Lambda$, causes acceleration in the expansion of the universe.

Further, one can introduce the density parameter, $\Omega$, as the ratio of the actual (or observed) density, $\rho$, to the critical

density, $\rho_{c}$, of the Friedmann universe. This ratio determines the overall geometry of the universe. It is well known that in the earlier models, which did not include a cosmological constant term, the critical density was regarded also as the watershed between an expanding and a contracting Universe. Recently, the critical density is estimated to be approximately five atoms (of monatomic hydrogen) per cubic meter, whereas the average density of ordinary matter in the Universe is believed to be 0.2 atoms per cubic meter [12]. Meanwhile, a much greater density comes from the unidentified dark matter. From the General Relativity, we know that both ordinary and dark matter contribute in favor of contracting of the universe. But, the largest part of density comes from the so-called dark energy that accounts for the cosmological constant term. Although the total density of the universe is equal to the critical density (exactly, up to measurement error), the dark energy does not lead to contraction of the universe. In fact, the dark energy contributes in favor of expanding of the universe.

Recently, the spatial geometry of the universe has been measured by the WMAP spacecraft. The result of that measuring showed that the spatial geometry of the universe is nearly flat. Following this result one can conclude that the universe can be well approximated by a model without the spatial curvature parameter $\mathrm{K}$ (i.e. $\mathrm{K}=0$ ). Meanwhile, this does not necessarily imply that the universe is infinite. It is because our measuring is related to the observation part of the universe that is limited, but the universe is much larger than the part we can see.

\section{EQUATIONS OF THE UNIVERSE MOTION IN AN ALPHA FIELD}

Here we show new approach to the description of the universe motion. This approach is based on the new Relativistic Alpha Field (RAF) theory [39,40,41]. This theory includes two non-dimensional field parameters $\alpha$ and $\alpha^{\prime}$ as functions of the space-time coordinates. Thus, for a gravitational potential field, the field parameters $\alpha$ and $\alpha$ have been identified by employing the Einstein's field equations in General Relativity [25,26,39].

It has been shown that the general nondiagonal form of the line element, $\mathrm{ds}^{2}$, of RAF theory, in the spherical polar coordinates, can be described by the equation:

$$
\begin{aligned}
& d s^{2}=-\alpha \alpha^{\prime} c^{2} d t^{2}-k\left(\alpha-\alpha^{\prime}\right) c d t d r \\
& +d r^{2}+r^{2} d \theta^{2}+r^{2} \sin ^{2} \theta d \phi^{2}, \quad v=\alpha \alpha^{\prime}, \lambda=k\left(\alpha^{\prime}-\alpha\right) / 2, \\
& d s^{2}=-v c^{2} d t^{2}+2 \lambda c d t d r+d r^{2}+r^{2} d \theta^{2}+r^{2} \sin ^{2} \theta d \phi^{2} .
\end{aligned}
$$

Here, $c$ is the speed of the light in a vacuum, $r$ is a radius vector, $\theta$ is an angle between radius vector $r$ and $z$-axis, and $\phi$ is an angle between projection of a radius vector $r$ on $(x-y)$ plane and $x$-axis. Parameter $\mathrm{k}= \pm 1$ (see [39]). The general solutions of that line element are presented by the relations:

$$
\begin{gathered}
\Lambda=\text { const. }, \rightarrow v=1-\frac{2 G M}{r c^{2}}-\frac{\Lambda}{3} r^{2}, \lambda=\sqrt{\frac{2 G M}{r c^{2}}+\frac{\Lambda}{3} r^{2}}, \\
\alpha_{1}=1-\sqrt{\frac{2 G M}{r c^{2}}+\frac{\Lambda}{3} r^{2}}, \alpha_{1}^{\prime}=1+\sqrt{\frac{2 G M}{r c^{2}}+\frac{\Lambda}{3} r^{2}}, \\
R A F T, \rightarrow v=1-\frac{2 G M}{r c^{2}}+\left(\frac{G M}{r c^{2}}\right)^{2}, \lambda=\sqrt{\frac{2 G M}{r c^{2}}-\left(\frac{G M}{r c^{2}}\right)^{2}}, \\
\alpha_{1}=1-\sqrt{\frac{2 G M}{r c^{2}}-\left(\frac{G M}{r c^{2}}\right)^{2}}, \quad \alpha_{1}^{\prime}=1+\sqrt{\frac{2 G M}{r c^{2}}-\left(\frac{G M}{r c^{2}}\right)^{2}} .
\end{gathered}
$$

Here RAFT means RAF theory, $G M / c^{2}$ is the Newton's constant of integration, $G$ is a gravitational constant, $M$ is a total gravitational mass, $r$ is a gravitational radius, $\Lambda$ is a cosmological constant and $c$ is the speed of the light in a vacuum. In RAF theory it is assumed that the cosmological constant $\Lambda=0$ and the energy momentum tensor $T_{\mu \eta} \neq 0$. If displacement four-vector $d X$ is defined in frame $\mathrm{K}$ by the expression: 
$d X \rightarrow K(c d t, d r, d \theta, d \phi)=\left\{d x^{i}\right\}, i=0,1,2,3$,

then the related covariant metric tensor of the line element (7) has the form [39]:

$$
\left[g_{\mu \eta}\right]=\left[\begin{array}{cccc}
-v & \lambda & 0 & 0 \\
\lambda & 1 & 0 & 0 \\
0 & 0 & r^{2} & 0 \\
0 & 0 & 0 & r^{2} \sin ^{2} \theta
\end{array}\right] .
$$

Here the no null components of the metric tensor $g_{\mu \eta}$ are given by the relations:

$$
\begin{aligned}
& \Lambda=\text { const. } \rightarrow g_{00}=-v=-\left(1-\frac{2 G M}{r c^{2}}-\frac{\Lambda}{3} r^{2}\right), \\
& g_{01}=g_{10}=\lambda=\sqrt{\frac{2 G M}{r c^{2}}+\frac{\Lambda}{3} r^{2}}, g_{11}=1, g_{22}=r^{2}, g_{33}=r^{2} \sin ^{2} \theta . \\
& R A F T \rightarrow g_{00}=-v=-\left(1-\frac{2 G M}{r c^{2}}+\left(\frac{G M}{r c^{2}}\right)^{2}\right), \\
& g_{01}=g_{10}=\lambda=\sqrt{\frac{2 G M}{r c^{2}}-\left(\frac{G M}{r c^{2}}\right)^{2}}, g_{11}=1, g_{22}=r^{2}, g_{33}=r^{2} \sin ^{2} \theta .
\end{aligned}
$$

The related determinant of the metric tensor (10) has the forms:

$$
\begin{gathered}
\operatorname{det}\left[g_{\mu \eta}\right]=-r^{4}\left(v+\lambda^{2}\right) \sin ^{2} \theta, r=1, \theta=\frac{\pi}{2}, \rightarrow \\
\operatorname{det}\left[g_{\mu \eta}\right]=-\left(v+\lambda^{2}\right)=-1, \quad v=1-\lambda^{2} .
\end{gathered}
$$

In the previous relation we use the normalization for $\mathrm{r}=1, \theta=$ $\pi / 2$, and the well known condition for the metric tensor of the line element det $\left(g_{\mu \eta}\right)=-1$. As the result we obtain the simple relation between field parameters $v$ and $\lambda$.

(a) Proposition 1. If the line element in an alpha field is defined by the relations (7) to (12) then the dynamic model of the Universe motion for $\Lambda=$ const. is given by the equations:

$$
\begin{aligned}
\Lambda=\text { const. } \rightarrow\left(\frac{\dot{r}}{r}\right)^{2} & +\frac{\mathrm{K} c^{2}}{r^{2}}-\frac{\Lambda c^{2}}{3}=\frac{8 \pi G}{3} \rho, \\
& \ddot{r}=-\frac{4 \pi G}{3}\left(\rho+\frac{3 p}{c^{2}}\right)+\frac{\Lambda c^{2}}{3} .
\end{aligned}
$$

Comparing the first equation in (13) with the first Friedmann equation in (4), and the second equation in (13) with the second Friedmann equation in (5), one can conclude that both equations have the same form as the Friedmann equations if we include the substitutions $r=\alpha, \dot{r}=\dot{\alpha}$ and $\ddot{r}=\ddot{\alpha}$. Thus, if the equations (13) describe the universe motion, then the radial coordinate $r(\mathrm{t})$ has the roll of the scale factor $\alpha(\mathrm{t})$.

The second equation in (13) states that both the energy density and the pressure cause a deceleration in the expansion of the universe. This is the consequence of gravitation, including that pressure is playing a similar role to that of energy (or mass) density. This is, of course, in accordance with the principles of general relativity. On the other side, the cosmological constant, $\Lambda$, causes acceleration in the expansion of the universe. In that case the cosmological constant, $\Lambda$, plays the role of the dark energy.

If the line element in an alpha field is defined by the relations (7) to (12) then the dynamic model of the Universe motion in RAF theory is given by the equations:

$$
\begin{array}{r}
R A F T \rightarrow\left(\frac{\dot{r}}{r}\right)^{2}+\frac{K c^{2}}{r^{2}}=\frac{8 \pi G}{3} \rho\left(1-\frac{2 \pi G}{3} \frac{\rho r^{2}}{c^{2}}\right), \\
\frac{\ddot{r}}{r}=-\frac{4 \pi G}{3}\left(\rho+\frac{3 p}{c^{2}}\right)\left(1-\frac{4 \pi G}{3} \frac{\rho r^{2}}{c^{2}}\right) .
\end{array}
$$

Comparing the first equation in (14) with the first Friedmann equation in (4), one can conclude that both equations have the same form of the two parts of the left side and the first part of the right side if the substitutions $r=\alpha$ and $\dot{r}=\dot{\alpha}$ are valid. Comparing the second equation in (14) with the second Friedmann equation in (5), one can conclude that both equations have the same form of the left side and of the first part of the right side if the substitutions $r=\alpha$ and $\ddot{r}=\ddot{\alpha}$ are valid. Thus, if the equations (14) describe the universe motion, then the radial coordinate $r(t)$ has the roll of the scale factor $\alpha(t)$. The first part of the right side of the second equation in (14) states that both the energy density and the pressure cause a deceleration in the expansion of the universe. This is the consequence of gravitation, including that pressure is playing a similar role to that of energy (or mass) density. This is, of course, in accordance with the principles of general relativity. On the other hand, the second part of the right side of the second equation in (14) causes acceleration in the expansion of the universe. This is the consequence of the positive (repulsive) gravitation force presented by RAF theory in $[39,40,41]$. This fact tells us that the positive (repulsive) gravitational force could be the source of the so called dark energy. Of course, this should be confirmed by the related experiments.

(b) Proof of the Proposition 1. In order to prove of the proposition 1 , for both cases $\Lambda=$ const. and in RAF theory, one can start with the Lagrangean of the line element (7):

$$
\begin{aligned}
& L=\left[-\frac{d s^{2}}{c^{2} d \tau^{2}}\right]^{1 / 2}=\frac{1}{c}\left[-g_{\mu \eta} \frac{d x^{\mu}}{d \tau} \frac{d x^{\eta}}{d \tau}\right]^{1 / 2}, \\
& \mu, \eta=0,1,2,3 \text {. }
\end{aligned}
$$

In the relation (15) $d \tau$ is the differential of the proper time $\tau$ and $\mathrm{dx}^{\mu}, \mathrm{dx}^{\eta}$, are components of the contravariant displacement four-vector $\mathrm{dX}$ in (9). Applying the equations from (7) to (11) and using (15) one obtains the Lagrangean in the following form:

$$
L=\left(\begin{array}{c}
\left.\nu\left(\frac{d t}{d \tau}\right)^{2}-\frac{1}{c^{2}} 2 \lambda c\left(\frac{d t}{d \tau}\right)\left(\frac{d r}{d \tau}\right)-\frac{1}{c^{2}}\left(\frac{d r}{d \tau}\right)^{2}-\right)^{1 / 2} . \\
-\frac{r^{2}}{c^{2}}\left(\frac{d \theta}{d \tau}\right)^{2}-\frac{r^{2}}{c^{2}} \sin ^{2} \theta\left(\frac{d \phi}{d \tau}\right)^{2}
\end{array}\right)^{2}
$$

The related Euler - Lagrange equations are given by the expressions: 


$$
\begin{aligned}
& \frac{\partial L}{\partial z^{i}}=\frac{d}{d \tau}\left(\frac{\partial L}{\partial \dot{z}^{i}}\right), \quad i=0,1,2,3, \\
& z^{o}=t, z^{1}=r, z^{2}=\theta, z^{3}=\phi, \dot{z}^{i}=\frac{d z^{i}}{d \tau} .
\end{aligned}
$$

Applying $\mathrm{i}=0$ to the relation (17) one obtains energy conservation equation:

$$
\begin{gathered}
\frac{\partial L}{\partial t}=0 \rightarrow \frac{\partial L}{\partial \dot{t}}=\text { const. }=\kappa \rightarrow \\
\quad \frac{d t}{d \tau}-\frac{\lambda}{c} \frac{d r}{d \tau}=\kappa, \frac{d \kappa}{d \tau}=\dot{\kappa}=0 .
\end{gathered}
$$

Here parameter $\kappa$ is the energy conservation constant. Applying $\mathrm{i}=3$ to the relation (17) one obtains angular momentum conservation equation:

$$
\begin{gathered}
\frac{\partial L}{\partial \phi}=0 \rightarrow \frac{\partial L}{\partial \dot{\phi}}=\text { const. }=h \rightarrow \\
-\frac{r^{2} \dot{\phi} \sin ^{2} \theta}{c^{2}}=h, \quad \frac{d h}{d \tau}=\dot{h}=0 .
\end{gathered}
$$

Parameter $h$ is the angular momentum conservation constant. In the case $\theta=\pi / 2$ (as in Newtonian theory) the angular momentum conservation equation (19) is transformed into the well-known relation:

$$
\begin{gathered}
\frac{\partial L}{\partial \phi}=0 \rightarrow \frac{\partial L}{\partial \dot{\phi}}=\text { const. }=h \rightarrow \\
-\frac{r^{2} \dot{\phi}}{c^{2}}=h, \quad \frac{d h}{d \tau}=\dot{h}=0 .
\end{gathered}
$$

Now, substituting the relations derived from (18) and (12), respectively:

$$
\frac{d t}{d \tau}=\left(\kappa+\frac{\lambda}{c} \frac{d r}{d \tau}\right) \nu^{-1}, \quad v=1-\lambda^{2},
$$

into the equation (16), and employing $\varepsilon=\mathrm{L}$ (where $\varepsilon=1$ for a time-like geodesics and $\varepsilon=0$ for a null geodesics) one obtains the following relation:

$$
\begin{aligned}
& \frac{1}{2}\left[\dot{r}^{2}+r^{2}\left(\dot{\theta}^{2}+\dot{\phi}^{2} \sin ^{2} \theta\right)\left(1-\lambda^{2}\right)\right] \\
& -\frac{\lambda^{2} \varepsilon^{2} c^{2}}{2}=\frac{c^{2}}{2}\left(\kappa^{2}-\varepsilon^{2}\right), \dot{r}=\frac{d r}{d \tau}, \dot{\theta}=\frac{d \theta}{d \tau}, \dot{\phi}=\frac{d \phi}{d \tau} .
\end{aligned}
$$

This relation represents the generalized energy equation of a particle with unit mass, where the sum of the kinetic energy $\mathrm{E}_{\mathrm{k}}$ and potential energy $\mathrm{E}_{\mathrm{p}}$ is equal to constant:

$$
\begin{aligned}
E_{k}= & \frac{1}{2}\left[\dot{r}^{2}+r^{2}\left(\dot{\theta}^{2}+\dot{\phi}^{2} \sin ^{2} \theta\right)\left(1-\lambda^{2}\right)\right], E_{p}=-\frac{\lambda^{2} \varepsilon^{2} c^{2}}{2}, \\
& \frac{c^{2}}{2}\left(\kappa^{2}-\varepsilon^{2}\right)=\text { const. }, \rightarrow E_{k}+E_{p}=\text { const } .
\end{aligned}
$$

For the case that parameter $\Lambda=$ constant, the relation (22) is transformed into the equation:

$$
\begin{gathered}
\Lambda=\text { const. } \rightarrow \lambda=\sqrt{\frac{2 G M}{r c^{2}}+\frac{\Lambda}{3} r^{2}}, \rightarrow \\
\frac{1}{2}\left[\dot{r}^{2}+r^{2}\left(\dot{\theta}^{2}+\dot{\phi}^{2} \sin ^{2} \theta\right)\left(1-\frac{2 G M}{r c^{2}}-\frac{\Lambda}{3} r^{2}\right)\right]- \\
-\frac{\left(\frac{2 G M}{r c^{2}}+\frac{\Lambda}{3} r^{2}\right) \varepsilon^{2} c^{2}}{2}=\frac{c^{2}}{2}\left(\kappa^{2}-\varepsilon^{2}\right) .
\end{gathered}
$$

If parameter $\Lambda=0$ and $\theta=\pi / 2$ (as in Newtonian theory), then the relation (24) can be transformed into the well-known Schwarzschild energy equation:

$$
\frac{1}{2}\left[\dot{r}^{2}+r^{2} \dot{\phi}^{2}\left(1-\frac{2 G M}{r c^{2}}\right)\right]-\frac{G M \varepsilon^{2}}{r}=\frac{c^{2}}{2}\left(k^{2}-\varepsilon^{2}\right) .
$$

Further, this relation can be reduced to the Newtonian energy equation by neglecting the term $2 \mathrm{GM} / \mathrm{rc}^{2}$ :

$\frac{1}{2}\left[\dot{r}^{2}+r^{2} \dot{\phi}^{2}\right]-\frac{G M \varepsilon^{2}}{r}=\frac{c^{2}}{2}\left(k^{2}-\varepsilon^{2}\right)$.

Thus, the energy equation (24) includes both Schwarzschild's and Newtonian energy equations as special approximations in a gravitational field.

In RAF theory the relation (22) is transformed into the new form of the generalized energy equation:

$$
\begin{gathered}
R A F T \rightarrow \lambda=\sqrt{\frac{2 G M}{r c^{2}}-\left(\frac{G M}{r c^{2}}\right)^{2}} \rightarrow \\
\frac{1}{2}\left[\dot{r}^{2}+r^{2}\left(\dot{\theta}^{2}+\dot{\phi}^{2} \sin ^{2} \theta\right)\left(1-\frac{2 G M}{r c^{2}}+\left(\frac{G M}{r c^{2}}\right)^{2}\right)\right] \\
-\frac{\left(\frac{2 G M}{r c^{2}}-\left(\frac{G M}{r c^{2}}\right)^{2}\right) \varepsilon^{2} c^{2}}{2}=\frac{c^{2}}{2}\left(\kappa^{2}-\varepsilon^{2}\right)
\end{gathered}
$$

This relation also represents that the sum of the kinetic energy $E_{k}$ and potential energy $E_{p}$ of a particle with unit mass is equal to constant:

$$
\begin{aligned}
& E_{k}=\frac{1}{2}\left[\dot{r}^{2}+r^{2}\left(\dot{\theta}^{2}+\dot{\phi}^{2} \sin ^{2} \theta\right)\left(1-\frac{2 G M}{r c^{2}}+\left(\frac{G M}{r c^{2}}\right)^{2}\right)\right], \\
& E_{p}=-\frac{\left(\frac{2 G M}{r c^{2}}-\left(\frac{G M}{r c^{2}}\right)^{2}\right) \varepsilon^{2} c^{2}}{2}, \frac{c^{2}}{2}\left(\kappa^{2}-\varepsilon^{2}\right)=\text { const } .
\end{aligned}
$$

If the quadratic term $\left(G M / r c^{2}\right)^{2}$ is neglected, and employing $\theta=\pi / 2$ (as in Newtonian theory), then the relation (27) is transformed into the Schwarzschild energy equation (25). 
Now, one can assume that the motion is in the radial direction, only. This means that $\dot{\theta}=0$ and $\dot{\phi}=0$. For that case, the generalized energy equation (22) is transformed into the form:

$$
\frac{\dot{r}^{2}}{2}-\frac{\lambda^{2} \varepsilon^{2} c^{2}}{2}=\frac{c^{2}}{2}\left(\kappa^{2}-\varepsilon^{2}\right) .
$$

For the case that parameter $\Lambda=$ constant, the relation (29) is transformed into the equation:

$$
\begin{aligned}
& \Lambda=\text { const. } \rightarrow \lambda=\sqrt{\frac{2 G M}{r c^{2}}+\frac{\Lambda}{3} r^{2}}, \rightarrow \\
& \frac{\dot{r}^{2}}{2}-\frac{\left(\frac{2 G M}{r c^{2}}+\frac{\Lambda}{3} r^{2}\right) \varepsilon^{2} c^{2}}{2}=\frac{c^{2}}{2}\left(\kappa^{2}-\varepsilon^{2}\right) .
\end{aligned}
$$

Now one can apply a mass density $\rho$, spatial curvature constant $\mathrm{K}$, and $\varepsilon=1$ (for a time-like geodesics):

$$
\frac{2 G M r^{2}}{r^{3} c^{2}}=\frac{8 \pi G}{3 c^{2}} \rho r^{2}, \quad\left(\varepsilon^{2}-\kappa^{2}\right)=\left(1-\kappa^{2}\right)=\mathrm{K} .
$$

By including the substitutions from (31), the second equation in (30) is transformed into the relation:

$$
\left(\frac{\dot{r}}{r}\right)^{2}+\frac{\mathrm{K} c^{2}}{r^{2}}-\frac{\Lambda c^{2}}{3}=\frac{8 \pi G}{3} \rho .
$$

Comparing this equation with the first Friedmann equation in (4), one can conclude that both equations have the same form and could be equal each to the other for substitution $r=\alpha$ and $\dot{r}=\dot{\alpha}$. Thus, if the equation (32) describes the universe motion, then the radial coordinate $r(t)$ has the roll of the scale factor $\alpha(t)$.

Applying a time derivative of (32) one obtains the following equation:

$$
\begin{gathered}
\ddot{r}=\frac{4 \pi G}{3} \frac{\dot{\rho}}{\dot{r}} r^{2}+\frac{8 \pi G}{3} \rho r+\frac{\Lambda c^{2}}{3} r, \rightarrow \\
\frac{\ddot{r}}{r}=\frac{4 \pi G}{3}\left(\dot{\rho} \frac{r}{\dot{r}}+2 \rho\right)+\frac{\Lambda c^{2}}{3} .
\end{gathered}
$$

In order to calculate a time derivative of the mass density $\dot{\rho}$, one should assume that the expansion of the universe is an adiabatic process. In that case, the thermo-dynamical approach can be considered. This is equivalent to the first law of thermodynamics and is resulting with the first equation in (6):

$$
\begin{gathered}
\dot{\rho}=-3 \frac{\dot{\alpha}}{\alpha}\left(\rho+\frac{p}{c^{2}}\right), \quad \alpha \rightarrow r, \quad \dot{\alpha} \rightarrow \dot{r}, \quad \rightarrow \\
\dot{\rho}=-3 \frac{\dot{r}}{r}\left(\rho+\frac{p}{c^{2}}\right) .
\end{gathered}
$$

Including the second equation from (34) into the second equation in (33) one obtains the following relation:

$$
\frac{\ddot{r}}{\mathrm{r}}=-\frac{4 \pi G}{3}\left(\rho+\frac{3 p}{c^{2}}\right)+\frac{\Lambda c^{2}}{3}
$$

Comparing this equation with the second equation in (5), one can conclude that both equations have the same form and could be equal each to the other for substitution $r=\alpha$ and $\ddot{r}=\ddot{\alpha}$. Thus, if the equation (35) describes the universe motion, then the radial coordinate $r(\mathrm{t})$ has the roll of the scale factor $\alpha(\mathrm{t})$. The equation in (35) states that both the energy density and the pressure cause a deceleration in the expansion of the universe. This is the consequence of gravitation, including that pressure is playing a similar role to that of energy (or mass) density. This is, of course, in accordance with the principles of general relativity. On the other side, the cosmological constant, $\Lambda$, causes acceleration in the expansion of the universe.

From the previous consideration one can take the equation from (32) and the equation from (35) and put them together:

$$
\begin{array}{r}
\Lambda=\text { const. } \rightarrow\left(\frac{\dot{r}}{r}\right)^{2}+\frac{\mathrm{K} c^{2}}{r^{2}}-\frac{\Lambda c^{2}}{3}=\frac{8 \pi G}{3} \rho, \\
\frac{\ddot{r}}{r}=-\frac{4 \pi G}{3}\left(\rho+\frac{3 p}{c^{2}}\right)+\frac{\Lambda c^{2}}{3} .
\end{array}
$$

The first relation in (36) is the velocity equation, while the second one is the acceleration equation. The both equations are valid for the case where parameter $\Lambda$ is a constant. Thus, the Proposition 1 , related to the case where $\Lambda=$ const. plays the role of the dark energy, is finished by the relations in (36).

In the case of RAF theory, the relation (29) is transformed into the equation:

$$
\begin{aligned}
& R A F T \rightarrow \lambda=\sqrt{\frac{2 G M}{r c^{2}}-\left(\frac{G M}{r c^{2}}\right)^{2}}, \rightarrow \\
& \frac{\dot{r}^{2}}{2}-\frac{\left(\frac{2 G M}{r c^{2}}-\left(\frac{G M}{r c^{2}}\right)^{2}\right) \varepsilon^{2} c^{2}}{2}=\frac{c^{2}}{2}\left(\kappa^{2}-\varepsilon^{2}\right) .
\end{aligned}
$$

Now one can apply a mass density $\rho$, spatial curvature constant $\mathrm{K}$, and $\varepsilon=1$ (for time-like geodesics):

$$
\begin{aligned}
& \frac{2 G M r^{2}}{r^{3} c^{2}}=\frac{8 \pi G}{3 c^{2}} \rho r^{2}, \quad \frac{G M r^{2}}{r^{3} c^{2}}=\frac{4 \pi G}{3 c^{2}} \rho r^{2}, \\
& \left(\frac{G M r^{2}}{r^{3} c^{2}}\right)^{2}=\left(\frac{4 \pi G}{3 c^{2}} \rho r^{2}\right)^{2},\left(\varepsilon^{2}-\kappa^{2}\right)=\left(1-\kappa^{2}\right)=\mathrm{K} .
\end{aligned}
$$

By including the substitutions from (38), the second equation in (37) is transformed into the relation:

$$
\left(\frac{\dot{r}}{r}\right)^{2}+\frac{\mathrm{K} c^{2}}{r^{2}}=\frac{8 \pi G}{3} \rho\left(1-\frac{2 \pi G}{3} \frac{\rho r^{2}}{c^{2}}\right) .
$$

Comparing this equation with the first Friedmann equation in (4), one can conclude that both equations have the same form of the two parts of the left side and the first part of the right side if the substitutions $r=\alpha$ and $\dot{r}=\dot{\alpha}$ are valid. Thus, if 
the equation (39) describes the universe motion, then the radial coordinate $r(t)$ has the roll of the scale factor $\alpha(t)$.

Applying a time derivative of (39) one obtains the following equation:

$$
\begin{gathered}
\ddot{r}=\left(\frac{4 \pi G}{3} \frac{\dot{\rho}}{\dot{r}} r^{2}+\frac{8 \pi G}{3} \rho r\right)\left(1-\frac{4 \pi G}{3} \frac{\rho r^{2}}{c^{2}}\right) \rightarrow \\
\frac{\ddot{r}}{r}=\frac{4 \pi G}{3}\left(\dot{\rho} \frac{r}{\dot{r}}+2 \rho\right)\left(1-\frac{4 \pi G}{3} \frac{\rho r^{2}}{c^{2}}\right) .
\end{gathered}
$$

In order to calculate a time derivative of the mass density $\dot{\rho}$, one should assume that the expansion of the universe is an adiabatic process. In that case, the thermo-dynamical approach can be considered. This is equivalent to the first law of thermodynamics and is resulting with the first equation in (6):

$$
\begin{gathered}
\dot{\rho}=-3 \frac{\dot{\alpha}}{\alpha}\left(\rho+\frac{p}{c^{2}}\right), \quad \alpha \rightarrow r, \quad \alpha \rightarrow \dot{r}, \quad \rightarrow \\
\dot{\rho}=-3 \frac{\dot{r}}{r}\left(\rho+\frac{p}{c^{2}}\right) .
\end{gathered}
$$

Including the second equation from (41) into the second equation in (40) one obtains the following relation:

$$
\frac{\ddot{\mathrm{r}}}{\mathrm{r}}=-\frac{4 \pi G}{3}\left(\rho+\frac{3 p}{c^{2}}\right)\left(1-\frac{4 \pi G}{3} \frac{\rho r^{2}}{c^{2}}\right) \text {. }
$$

Comparing this equation with the second equation in (5), one can conclude that both equations have the same form of the left side and of the first part of the right side if the substitution $r=\alpha$ and $\ddot{r}=\ddot{\alpha}$ are valid. Thus, if the equation (42) describes the universe motion, then the radial coordinate $r(\mathrm{t})$ has the roll of the scale factor $\alpha(t)$. The equation (42) states that the first part of the right side causes a deceleration in the expansion of the universe. On the other hand, the second part of the right side causes acceleration in the expansion of the universe.

From the previous consideration one can take the equation from (39) and the equation from (42) and put them together:

$$
\begin{aligned}
R A F T \rightarrow\left(\frac{\dot{r}}{r}\right)^{2} & +\frac{\mathrm{K} c^{2}}{r^{2}}=\frac{8 \pi G}{3} \rho\left(1-\frac{2 \pi G}{3} \frac{\rho r^{2}}{c^{2}}\right), \\
\frac{\ddot{r}}{r} & =-\frac{4 \pi G}{3}\left(\rho+\frac{3 p}{c^{2}}\right)\left(1-\frac{4 \pi G}{3} \frac{\rho r^{2}}{c^{2}}\right) .
\end{aligned}
$$

The first relation in (43) is the velocity equation, while the second one is the acceleration equation. The both equations are valid in RAF theory. Thus, the Proposition 1, related to RAF theory [39,40,41], where positive (repulsive) force plays the role of the dark energy, is finished by the relations in (43). This fact tells us that the positive (repulsive) gravitational force could be the source of the so called dark energy. Of course, this should be confirmed by the related experiments.

The presented results in RAF theory can also be interpreted by the assumption that the so called cosmological constant is the function of gravitational radius, $\Lambda=f(r)$, (see [35]).

\section{CONCLUSION}

The new derivations of the dynamic equations of the universe motion, for the cases $\Lambda=$ constant and RAF theory approach, are proposed. These equations are compared with the well known Friedmann equations. In the model with $\Lambda=$ constant the acceleration in the expansion of the universe is caused by parameter $\Lambda$. On the contrary, in the model obtained by RAF theory approach, the gravitational positive (repulsive) force causes acceleration in the expansion of the universe. In the Relativistic Alpha Field (RAF) theory $[39,40,41]$, one of the predictions is that the gravitational force becomes positive (repulsive) if $\left(G M / r c^{2}\right)>1$, that could be a source of a dark energy. Here derived equations of the universe motion show that positive (repulsive) gravitational force really can produce an accelerating rate of the universe motion and therefore could be a candidate for the source of a dark energy. In that sense a dark energy could be seen as a positive (repulsive) gravitational energy.

\section{REFERENCES}

[1] A. Friedmann, Über die Krümmung des Raumes. Z.Phys. 10 (1), pp. 377-386 (in German), (1922). A. Friedman, On the Curvature of Space. General Relativity and Gravitation 31 (12), pp. 1991-2000 (in English), (1999)

[2] A. Friedmann, Über die Möglichkeit einer Welt mit konstanter negativer Krümmung des Raumes. Z.Phys. 21 (1), pp. 326-332 (in German), (1924). A. Friedman, On the Possibility of a World with Constant Negative Curvature of Space. General Relativity and Gravitation 31 (12), pp. 2001-2008 (in English), (1999).

[3] G.Lemaître, Expansion of the Universe, a Homogeneous Universe of Constant Mass and Increasing Radius Accounting for the Radial Velocity of Extra Galactic Nebulae. Monthly Notices of the Royal Astronomical Society 91, pp. 483-490, (1931).

[4] G. Lemaître, L'Univers en Expansion. Annales de la Société Scientifique de Bruxelles A53, pp. 51-85 (in French), (1933).

[5] H.P. Robertson, Kinematics and World Structure. Astrophysical Journal 82, pp. 284-301, (1935).

[6] H.P. Robertson, Kinematics and World Structure II. Astrophysical Journal 83, pp. 187-201, (1936).

[7] H.P. Robertson, Kinematics and World Structure III. Astrophysical Journal 83, pp. 257-271, (1936).

[8] A. G. Walker, On Milne's Theory of World Structure. Proceedings of the London Mathematical Society 242 (1), pp. 90-127, (1937).

[9] L. Bergström, A. Goobar, Cosmology and Particle Astrophysics. Sprint, p. 61, $2^{\text {nd }}$ ed., ISBN 3540329242, (2006).

[10] A. Einstein, The Meaning of Relativity, 5th ed. ( Princ. Univ. Press, Princet., N. J., 1955).

[11] Ray d'Inverno, Introducing Einstein's Relativity, (Clarendon Press, 394 p., ISBN 0198596537, 1992).

[12] M. Rees, Just Six Numbers, (Orion Book, London, p. 81, p. 82., 2000)

[13] M. Lachieze-Rey, J.P. Luminet, Cosmic Topology. Physical Reports 254 (3), pp. 135-214, (1995).

[14] G.F.R. Ellis, H. van Elst, Cosmological Models. In Marc Lachieze-Rey. Theoretical and Observational Cosmology. (NATO Science Series C. 541, pp. 1-116, 1999).

[15] P. Ojeda, H. Rosu, Supersymmetry of FRW Barotropic Cosmologies, International Journal of Theoretical Physics 45 (6), pp. 1191-1196. (2006).

[16] B, Tersic, Lectures Notes on Astrophysics. http://nicadd.niu.edu/ btersic/PHYS652_notes.pdf, Retrieved 20 July (2011).

[17] Wikipedia, the free encyclopedia. (2012). FLRW Metrics. http://en.wikipedia.org/wiki/Friedmann_Lemaitre_Robertson_Walker Metrics, Retrieved 19 March (2012)

[18] Wikipedia, the free encyclopedia. (2012). Friedmann Equations. http://en.wikipedia.org/wiki/Friedmann equations , Retrieved 19 March (2012).

[19] A.H.Guth, Inflationary Universe: A Possible Solution to the Horizon and Flatness Problems. Phys. Rev. D23, 347 (1981); New York Academy of Sciences Magazine, Jan./Febr., (2002). 
[20] S. Perlmutter et al., Nature 391, 51 (1998); Riess A. et al., Astron. J. 1161009 (1998).

[21] R.R. Caldwell et al., Phys. Rev. Lett. 80, 1582 (1998), Caldwell R. R., astro- ph/9908168.

[22] L. Parker, A. Raval, Phys. Rev. D 60, 063512 (1999) ; 60, 123502 (1999) ; 62, 083503 (2000).

[23] P.J. Steinhard, N. Turok, http://feynman.princeton.edu/ steinh/, hepth/ 0111030, Science, in the press (2002), hepth/0111098, Phys. Rev. D, in the press (2002).

[24] B. Novakovic, D. Novakovic, A. Novakovic, A New General Lorentz Transformation Model. AIP Conf. Proc., CP 517, (2000), CASYS'99-Third Int. Conf., ed. by D. M. Dubois, pp. 437-450, Liege, Belgium (1999).

[25] B. Novakovic, D. Novakovic, A. Novakovic, A Metric Tensor of the New General Lorentz Transformation Model. CASYS Int. Journal of Comp. Anticipatory Systems, ed. by D. M. Dubois, Publ. by CHAOS, Liege, Belgium, Vol. 10, pp. 199-217. (2001).

[26] B. Novakovic, D. Novakovic, A. Novakovic, (2004). The Cosmological Constant $\Lambda$ is not Really Constant but the Function of a Gravitational Radius. AIP Conf. Proc. CP718, American Institute of Physics, ed. by D. M. Dubois, pp.133-143. (2004).

[27] B. Novakovic, D. Novakovic, A. Novakovic, A new Approach to Unification of Potential Fields Using GLT Model. CASYS Int. Journal of Comp. Anticipatory Systems, ed. by D. M. Dubois, Publ. by CHAOS, Liege, Belgium, Vol. 11, pp. 196-211, (2002).

[28] B. Sterling, Surprise! Our Little Corner of the Universe is Even Smaller than we Thought, (2003). www.wired.com/wired/archive/11.11/view.html?pg =4.

[29] G.M. Kremer, Cosmological Models Described by a Mixture of der Waals Fluid and Dark Energy. Phys. Rev. D 68, 123507 (2003).

[30] S.E. Jorás, T.J. Stuchi, Chaos in a Closed Friedmann Robertson Walker Universe: An Imaginary Approach. Phys. Rev. D 68, 123525 (2003).

[31] M. Susperregi, Dark Energy and Dark Matter from an Inhomogeneous Dilaton. Phys. Rev. D 68, 123509 (2003).

[32] P.G. Castro, M. Douspis, P.G. Ferreira, Scale of Homogeneity of the Universe from WMAP. Phys. Rev. D 68, 127301 (2003).

[33] P.P. Avelino, C. J.A.P. Martins, C. Santos, E.P.S. Shellard, Topological Defects: A Problem for Cyclic Universe. Phys. Rev. D 68, $123502(2003)$

[34] J. Khoury, P.J. Steinhardt, N. Turok, Designing Cyclic Universe Models. Phys. Rev. Lett. 92, 031302 (2004).

[35] B. Novakovic, D. Novakovic, A. Novakovic, A New Dynamic Model of the Universe Motion. CASYS Int. Journal of Comp. Anticipatory Systems, ed. by D. M. Dubois, Publ. by CHAOS, Liege, Belgium, Vol. 16, pp. 147-162 (2004).

[36] A.D. Aczel, God's Equation. Introduction, v $=0.956$ c, p. 11. Izvori, Zagreb, (2001).

[37] J. E. Lidsey, Physical Review D 70, 041302 (2004).

[38] T. Clifton, C. Clarkson, P. Bull, Isotropic Blackbody Cosmic Microwave Background Radiation as Evidence for a Homogeneous Universe. Phys. Rev. Lett. 109, 051303 (2012).

[39] B. M. Novakovic, Relativistic alpha field theory - Part I. International Journal of New Technology and Research, Vol.1, No.5, pp.23-30, IJNTR01050015, (2015).

[40] B. M. Novakovic, Relativistic alpha field theory - Part II. International Journal of New Technology and Research, Vol.1, No.5, pp.31-38, IJNTR01050016, (2015).

[41] B. M. Novakovic, Relativistic alpha field theory-Part III. International Journal of New Technology and Research, Vol.1, No.5, pp.39-47, IJNTR01050017, (2015).

[42] A. Pisani, et al., Counting voids to probe dark energy, Phys. Rev. D 92, 083531 (2015).

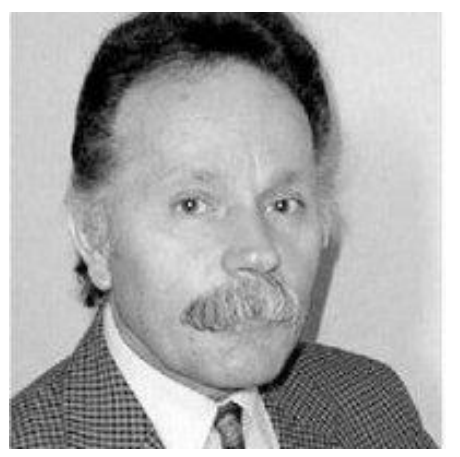

Branko Novakovic is a Professor emeritus at FSB - University of Zagreb, Croatia. Prof Novakovic received his $\mathrm{PhD}$ from the FSB-University of Zagreb in 1978. His research interests include physics, control systems, robotics, neural networks, and fuzzy control. He is author of two books, Control Systems (1985) and Control Methods in Robotics, Flexible Manufacturing Systems and Processes (1990), and the first co-author of a book Artificial Neural Networks (1998). He has published over 220 research papers in his research of interest. 